DOI: https://doi.org/10.30837/MMP.2021.241

\title{
STUDY OF STRUCTURAL RELATIONSHIPS AND MODELS OF INFORMATION SUPPORT FOR ENERGY LIFE CYCLE MANAGEMENT IN MICROGRID
}

Shendryk V., Parfenenko Yu., Pavlenko P., Boiko O., Shendryk S.

The study presents an analysis of the structural relationships of complex energy systems based on renewable energy sources and information processes that support their work. Modeling of information support of energy life cycle management in Microgrid is performed. First, the structural models of the life cycle of energy and information in Microgrid are considered, the analysis of information processes in the management of energy infrastructure. It is determined which models of process forecasting in energy systems and models of decision support in energy life cycle management in Microgrid have the greatest efficiency.

\section{INTRODUCTION}

In recent years, according to the International Climate Agreement [1], countries need to reduce greenhouse gas emissions and introduce the transition to a low-carbon, sustainable economy. Achieving these goals is possible through the implementation of energy efficiency strategies and energy production from renewable energy sources (RES), restructuring of energy systems taking into account the active use of RES.

Since most RES can be used in a decentralized manner, the distributed principle of power generation and micro-grid (MG) is a good development and integration option for these systems.

Due to their distributed structure, Microgrid networks are heterogeneous and complex systems that are equipped with a wide range of energy generation elements and are used by consumers of various types from industrial to domestic. Because the level of energy generation from renewable sources is difficult to predict and depends on meteorological conditions, Microgrid networks require the use of advanced control and adaptive control to fully realize their potential and ensure balance in the 
energy network [2]. The task of expanding the existing Microgrid networks and creating Microgrid clusters with decentralized management is urgent, which requires the development of appropriate information support.

The growing number of measuring and control devices owned by Microgrid networks, as well as the challenges of improving the quality of Microgrid networks, the development of the Microgrid concept as a component of Energy 4.0 in Industry 4.0, necessitated the development of models and tools for continuous monitoring, processing and analysis data at different stages of the Microgrid life cycle.

Therefore, it is important to determine the typical structure of the energy system, analyse the life cycle of energy and information in it, analyse information processes in planning and management of energy infrastructure, identify models for forecasting and decision support in energy lifecycle management in Microgrid.

\section{STRUCTURAL MODELS OF THE LIFE CYCLE OF ENERGY AND INFORMATION IN MICROGRID}

Microgrid can be defined as a network of electricity distribution with its own energy resources, which is able to operate autonomously as a small electrical network [3]. Such networks are specially designed to operate in the low or medium voltage range using renewable energy sources such as solar panels and / or wind turbines, fuel cells, etc. and battery storage systems to meet local load requirements. Microgrid can operate in isolation or be connected to the main network through a macro station - a subscriber station. These characteristics make them suitable for use in rural or remote areas.

Along with the growing development of renewable energy, the concept of multi-microgrids has emerged, which describes the idea of a cluster of interconnected microgrids. The purpose of this connection is to achieve firmness and stability through energy exchange and mitigate the impact of connecting distributed generation units to power systems.

This paper considers a multi-microgrid that covers several microgrids. Each Microgrid can consist of several wind turbines and/or solar panels (EG), a battery 
storage system (BESS), and local loads. All microgrids are connected to a distribution network, which is connected to the main network (Main GRID) at the point of joint connection. There is a connection between each of the networks both at the energy and information level. Through the measuring elements, each micronetwork can receive the provided data from the distribution network operator (DNO) and has the opportunity to share its own information with DNO or other micronetworks. In this case, energy can be transmitted bilaterally between the DNO and each microgrid. Meanwhile, the DNO can exchange energy bilaterally with the main network. A typical scheme of such a system is shown in fig. 1.1.

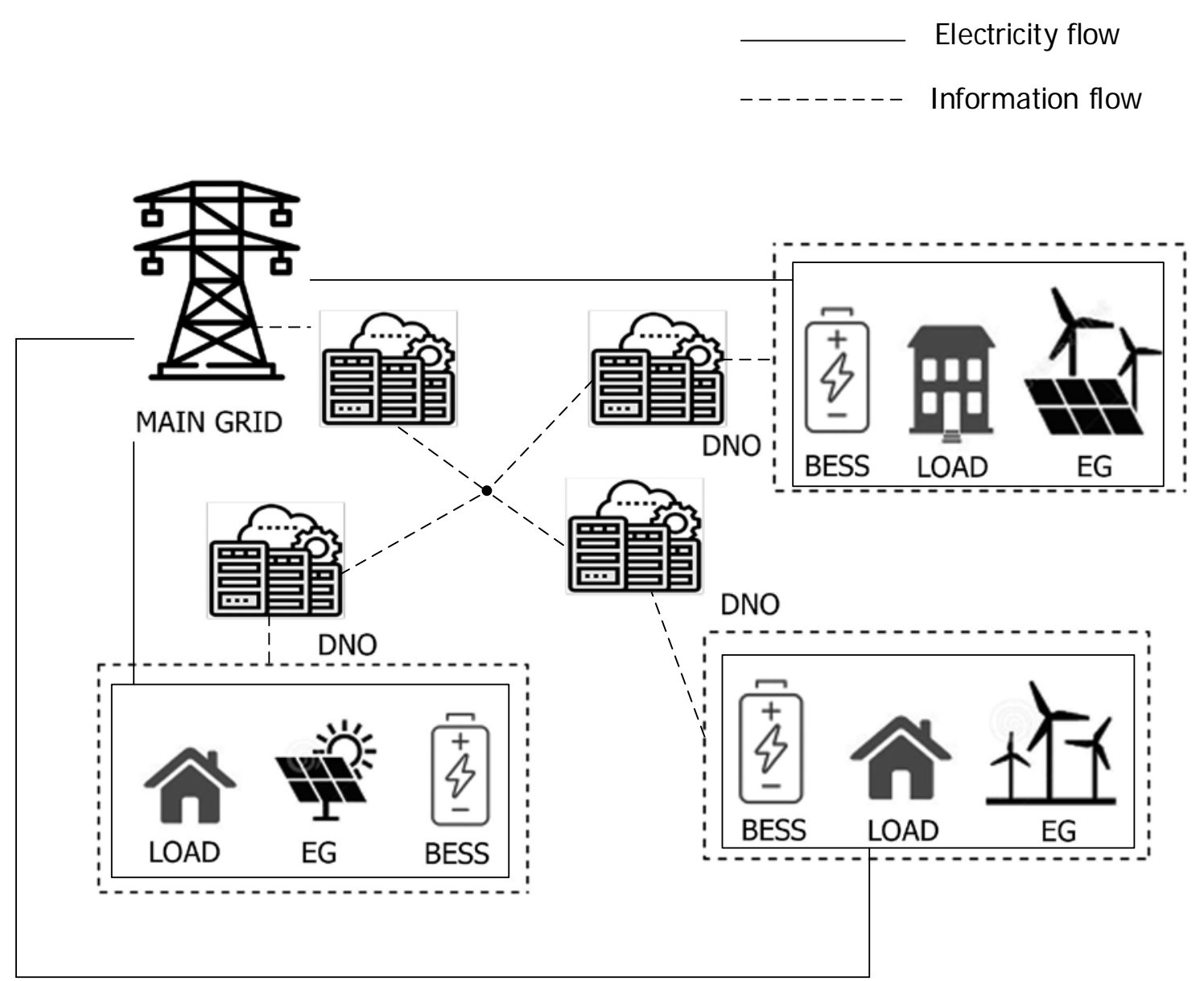

Fig. 1.1. A typical multi-microgrid scheme

The large number of different generation systems that make up the multimicrogrid can have a negative impact on the power supply due to fluctuations in voltage and power quality due to uncertainty in the generation process. For example, 
weather conditions affect the energy potential of solar and wind power generation, and load needs can lead to energy shortages or surpluses, both at different times of the day and at different times of the year. Therefore, while distributed energy resources can potentially reduce the need for traditional energy system expansion, new challenges arise in ensuring the efficient operation, monitoring and control of microgrids.

To solve them, various approaches can be used: the use of storage batteries to mitigate the peaks formed in these systems; use of intermittent renewable energy management models; stochastic methods of energy management in several microsets; demand response mechanisms based on consumer forecasts, etc.

Within one Microgrid, there are electric and information flows that need to be effectively controlled. The fundamentals of information management are presented in Krcmar [4]. The authors define three layers of data that are relevant for information management:

- $\quad$ the lower level determines the management of technical infrastructure, namely information and communication technologies.

- $\quad$ the second level focuses on the management of information systems (e.g., data structure).

- $\quad$ the upper level deals with the tasks of managing the results of systems. For example, the use of information to improve the transmission and distribution of electricity.

In [5] it is proposed to consider Microgrid not only as energy technologies for the production, storage, distribution and consumption of energy, but also with a focus on the information component. Microgrid as a structure should be considered in addition to political, economic, social, technological, legal and environmental factors. It is assumed that the Microgrid can operate in the mode of connection to an external electrical network or in a stand-alone, island mode.

The information flow in Microgrid allows the exchange of information between neighbouring networks and, for example, weather forecast, measurement 
data or trade in external energy markets [6]. Flows from the information network can affect the physical flows in the micro grid: for example, information about the price of electricity affects the consumption or sale of electricity. External parameters, such as market characteristics of the equipment, affect the design of micronetworks.

Fig. 1.2 shows a typical organization of the power grid system taking into account information flows.

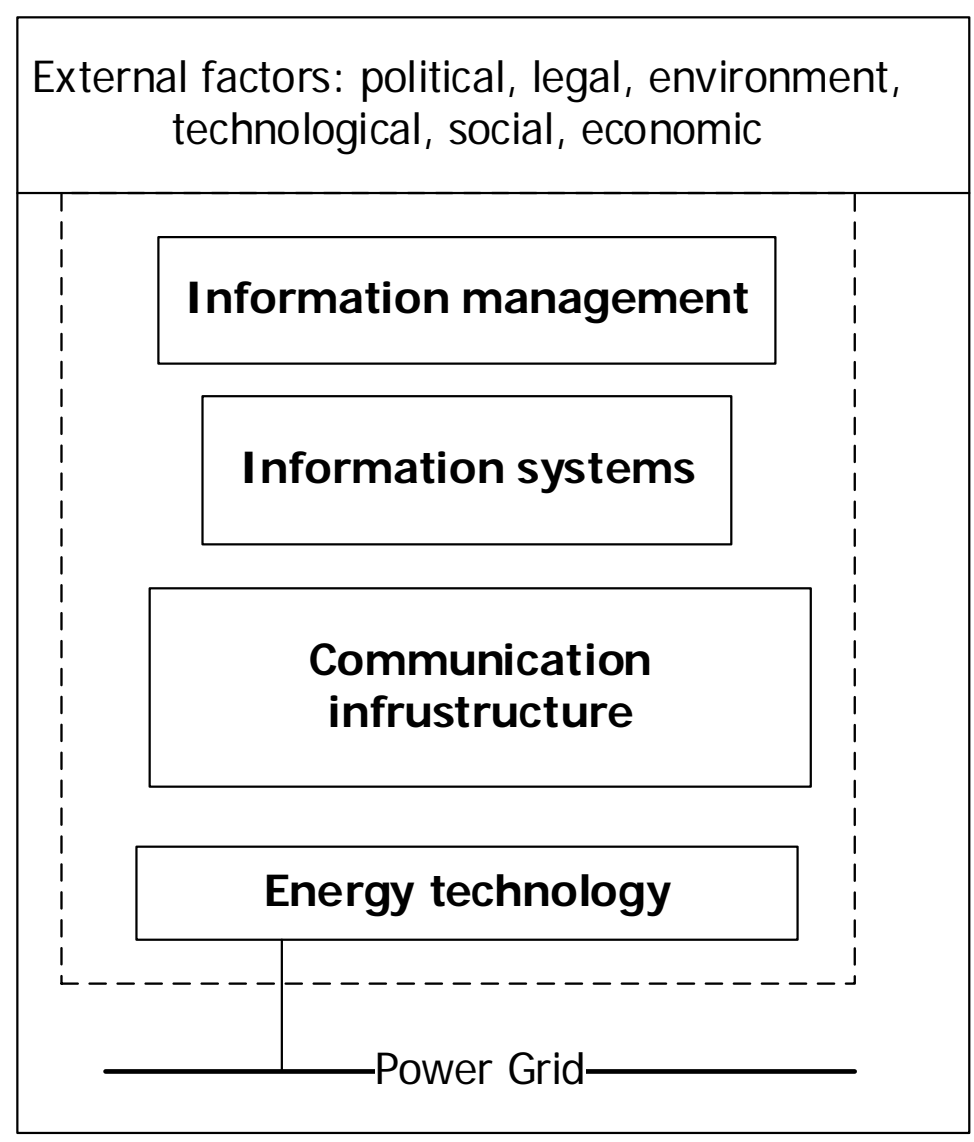

Fig. 1.2. Information levels of microgrid operation

The following levels can be distinguished: I level of Energy Technologies, II level of Information and Communication Infrastructure, located above level I, which means that the basic IT is used for effective management of energy technologies of micronetworks. Level III - Information Systems, which may contain applications for control, planning and administration in the context of micronetworks. The upper IV level of "Information Management" considers management tasks, for example, matching demand with supply. 
According to the proposed scheme, certain information processes take place at each level, which must be determined in advance when managing energy infrastructure facilities.

\section{ANALYSIS OF INFORMATION PROCESSES IN THE MANAGEMENT OF ENERGY INFRASTRUCTURE}

The process of transition from planning to implementation and management of the complex of energy networks takes place step by step. An example of such a process can be presented as a diagram in fig. 2.1.

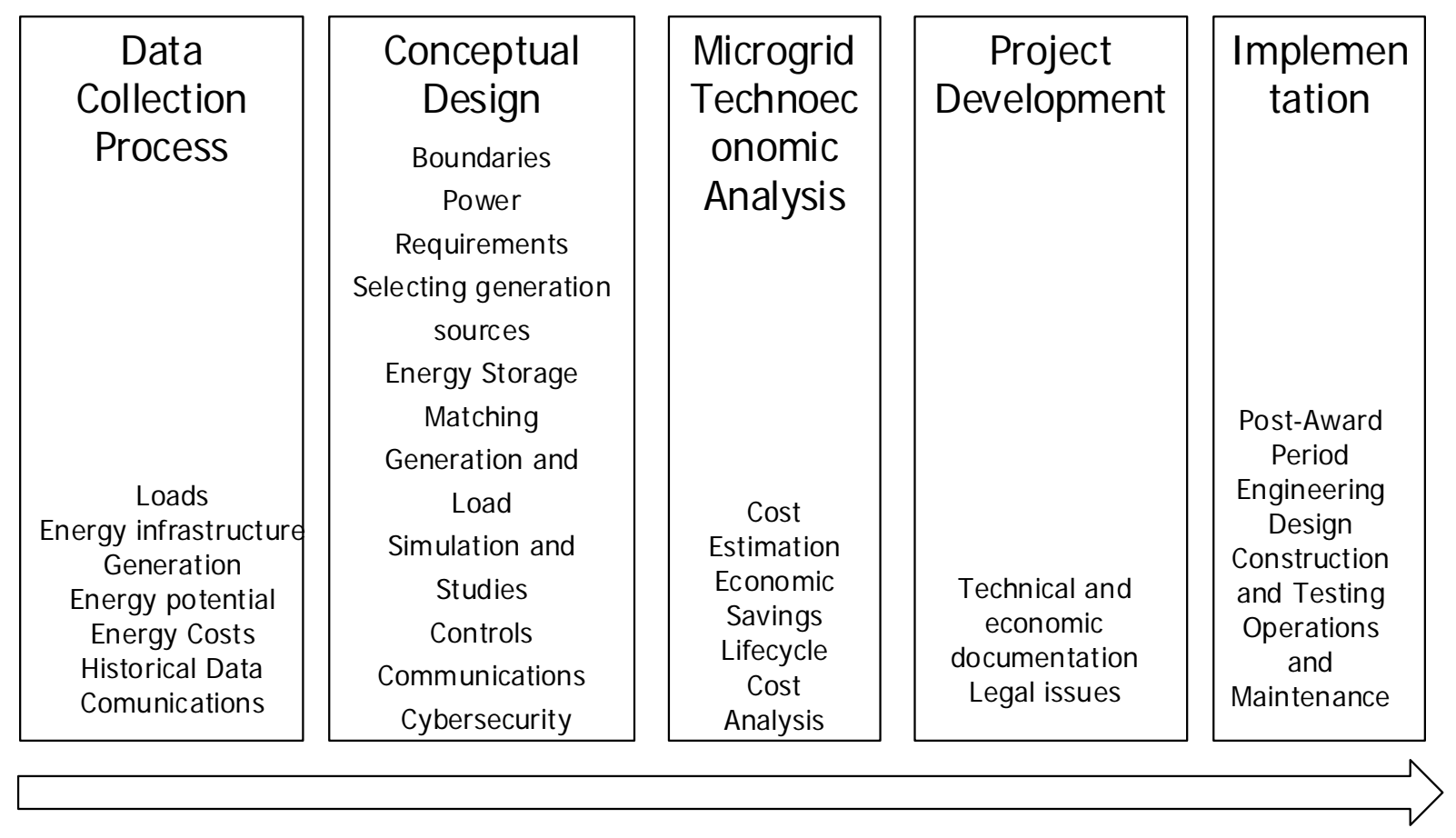

Fig. 2.1. Microgrid implementation process

Data Collection Process. This phase aims to gather information for a better understanding of loads, energy distribution, existing systems, production resources, energy costs and the historical sustainability of typical facilities. It is necessary to obtain information on the magnitudes and trends of possible energy loads, existing systems in the area, possible sources of energy generation, the presence of economic incentives, and the actual cost of electricity at the site, maps of power lines, grid control systems, and more. 
Conceptual Design. At this stage, system requirements are formed, the existing conditions defined in the previous step are analysed. The conceptual design must be formed in accordance with these constraints.

It is necessary to determine the power requirements, select the sources of generation and storage of energy, to analyse the compliance of generation and load. Also at this stage it is often necessary to simulate the operation of the network. It is important to define the microcontroller, communication and cybersecurity controls.

Microgrid Technoeconomic Analysis. Carrying out a technical and economic assessment, which analyzes the capital costs of the system, savings opportunities and financial returns.

Project Development. Preparation of technical and economic documentation, coordination of legal issues.

Implementation. At this stage, it is necessary to develop, build, commission and test the project to ensure effective operation.

Modern development of Microgrid networks is impossible without the introduction of information and communication technologies to achieve the tasks described above. The use of information systems as an intelligent superstructure over the communication infrastructure, which implements the measurement of energy networks and their management, is the main direction of development of energy management system Microgrid, aimed at improving energy efficiency and balancing electricity generation and consumption.

Compared to traditional power grids, the developed information and communication infrastructure provides arrays of data that can be collected and processed in real time. It is subject to the requirements of reliability, safety, scalability and ease of management [7].

Components of the information and communication infrastructure of the Microgrid network are sensors for remote monitoring of network parameters, distributed automatic control devices. The information and communication infrastructure that provides support for Microgrid management on the consumer side 
includes devices for monitoring electrical load, voltage measurement, automatic measuring devices, and meters.

The information and communication infrastructure exchanges data with the Microgrid computing platform, which is a layer of operational management at the system level. The computing platform consists of servers that support all processes of support for operational management of Microgrid, such as data collection from sensors in real time, implementation of network optimization and mode switching, Microgrid network scheduling, rapid response to changes in electricity demand, SCADA data analysis, correction of failures in the mode of operation of the network, automation of transmission and distribution of energy. The computing platform can be centralized or distributed, built on an agent approach.

The link between the information and communication infrastructure and the automated network management system Microgrid is a set of software that implements various tasks of information support lifecycle management of the Microgrid network from its design to operation, the main of which are listed below:

- planning the structure of the Microgrid network, including the selection of the optimal location of energy generation elements and their configuration parameters;

- forecasting the demand for electricity, price tariffs and generation opportunities;

- optimization of the power grid;

- support for energy management decision-making;

- providing data display for the end user in the web or mobile interface.

The components of the main activities that ensure the implementation of the Microgrid concept - design and operational management were analyzed. The activity diagram of the Microgrid network design process is shown in fig. 2.2. 


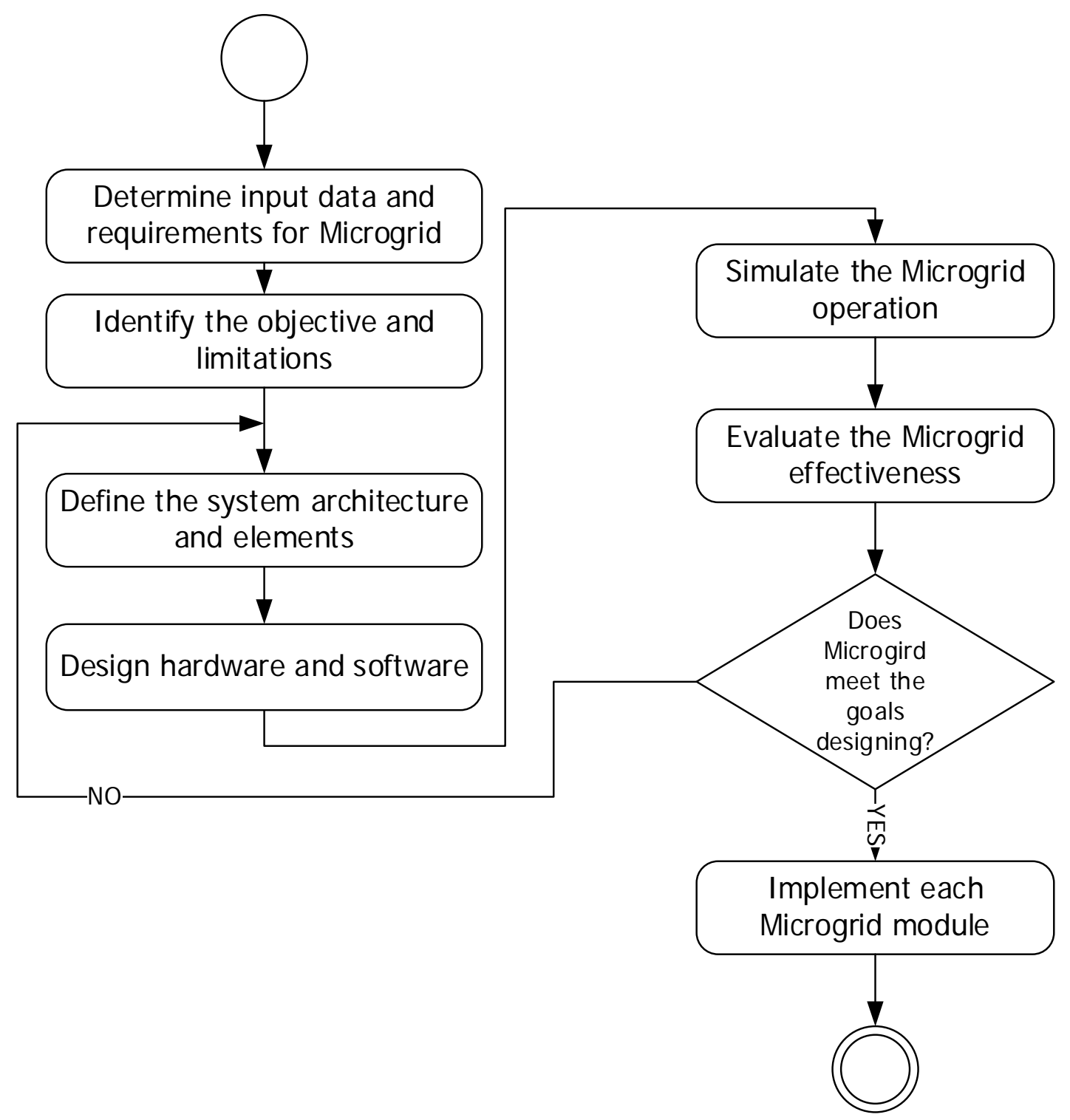

Fig. 2.2. Activity diagram of the Microgrid design process

The main subprocesses of designing a Microgrid structure are defining goals, overall architecture, choosing the optimal Microgrid location if there are alternatives, configuring key elements, defining the architecture and software of the Microgrid network, and verifying that the designed architecture conforms to the goals. The diagram of activity of process of operative management of the Microgrid network in a normal mode within one day is shown in fig. 2.3 [8]. 


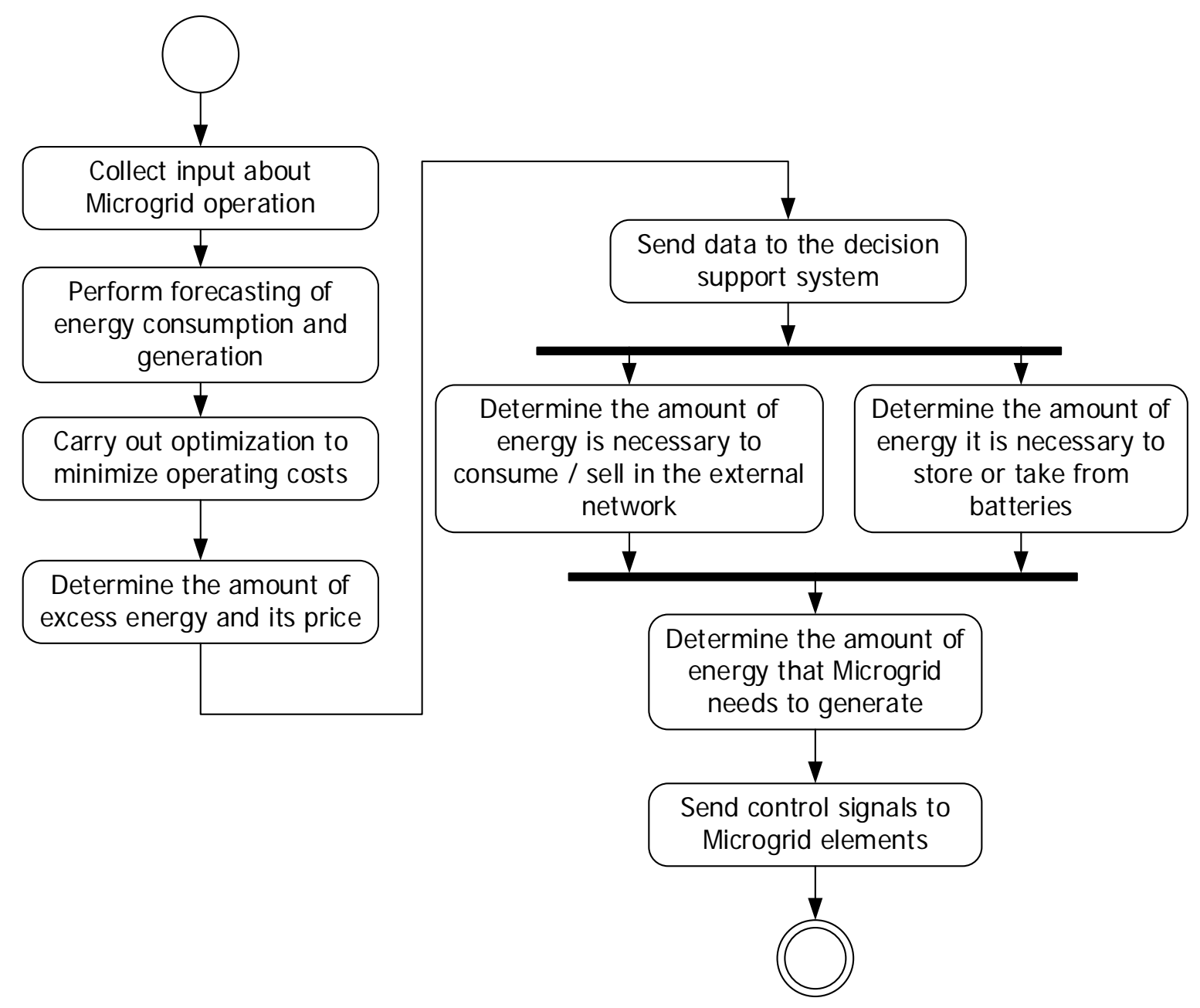

Fig. 2.3. Microgrid online management activity diagram in normal mode

In the operational management of the Microgrid network, such subprocesses are performed as monitoring of network and environmental data, forecasting electricity needs and possibilities of its generation from distributed sources, determining the amount of excess or shortage of generated energy and possible power source, optimizing network operation, Based on all the data from the previous stages, decisions are made on the regulation of network power, the amount of energy generated, determines the amount of excess energy that can be supplied to the centralized network, control signals are sent to the appropriate devices that control the Microgrid network. 
The above processes are supported by the Microgrid energy management system [9, 10], the general architecture of which is shown in fig. 2.4.

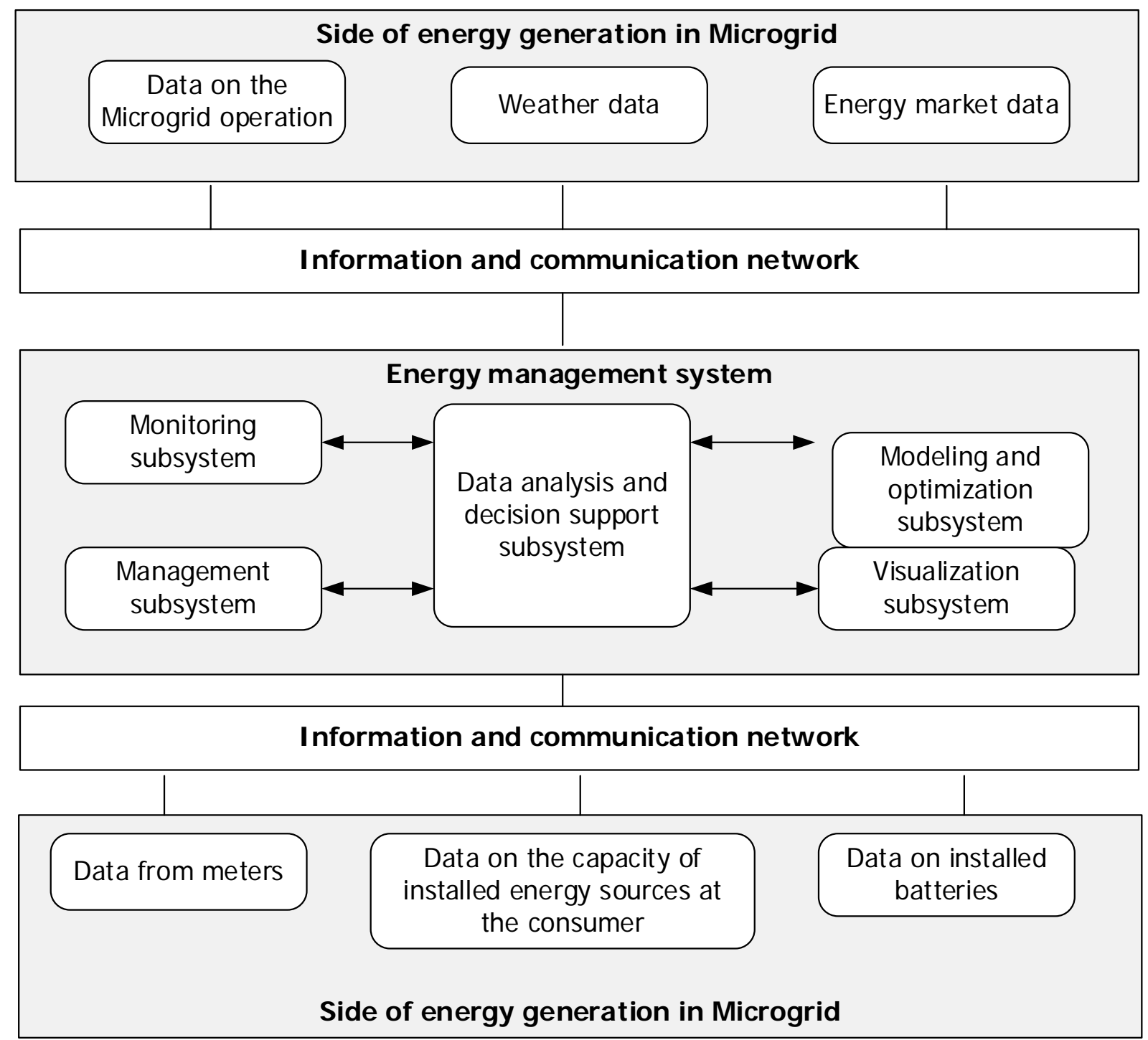

Fig. 2.4. General architecture of the Microgrid energy management system

Fig. 2.4 shows that information support for Microgrid facility management is provided through models of monitoring, optimization, data analysis, forecasting and developed appropriate software.

Monitoring provides real-time data collection, verification and pre-processing of data, establishment of deviations from regulatory parameters, entry into the 
database of operational data and aggregation of data at certain time intervals. The Microgrid performance monitoring model includes a set of data characterizing the current technical condition of the Microgrid and the main indicators of its operation, a set of meteorological conditions in the area where the Microgrid is installed, a set of factors influencing electricity demand, such as business schedule or factor seasonality, a set of forecast data of meteorological conditions. Data collection and processing can be performed using software agent technology that retrieves data according to a set schedule, for example, once an hour, or in the event of an event, such as a deviation from the set mode of operation of Microgrid. To accumulate monitoring data, it is advisable to use cloud platforms, such as Microsoft Azure, IBM Cloud, etc.

Methods of linear and nonlinear programming, dynamic and stochastic programming, metaheuristic algorithms, multiagent and intelligent technologies are used to solve optimization problems. Due to the complexity of the structure of Microgrid and the peculiarities of its work, instead of classical optimization techniques, dynamic programming is used, which allows to discretize optimization problems and divide them into subtasks. Metaheuristic algorithms allow finding mechanisms for achieving optimal operation and control of micronetwork energy using genetic algorithms and algorithms of biological evolution. Stochastic programming methods are used as a method of detecting network elements with possible deviations in the parameters of operation in the future, often used in the subsystems of storage of electricity.

The multi-agent optimization methods used in Microgrid allow distributed micronet management by agents that have autonomous behaviour and perform optimization tasks for various Microgrid elements, such as power generators and storage systems for specific purposes. The advantage of the agent approach is that the software agents communicate with each other and can adjust their own optimization goals depending on the set criterion for optimizing the performance of MicroGrid as a whole. 
Forecasting is used to determine the performance of the Microgrid network for certain periods of time in the future, as well as to determine changes in network impact factors, such as climatic conditions, that affect the level of electricity generation from renewable sources. The following section analyses the effectiveness of Microgrid's energy consumption forecasting models.

\section{MODELS OF PROCESS FORECASTING IN ENERGY SYSTEMS}

The work of Microgrid is characterized by uncertainty, it cannot be formalized by a mathematical relation, so when studying the work of the network, forecasting techniques are widely used. Forecasting is most often used to solve such tasks in Microgrid as planning the needs for energy resources and the level of their generation, determining the peak periods of energy consumption, determining the possible failure of installed equipment, determining prices for energy resources in the future. The use of forecast data is used both in making operational decisions to optimize the load on the network and in making strategic decisions by energy market participants. This enhances the reliability of the power grid.

Forecasting in Microgrid according to the periods of time for which the forecast is given is divided into the following categories [11]:

- long-term - for a period of one to several years, used in strategic planning, network expansion by adding new energy sources, connecting new consumers;

- medium-term - for the period from a week to a year, used for planning the maintenance of the network, the cost of purchasing fuel, estimating the profit from the sale of electricity to the general network;

- short-term - for a period of one hour to a week, used to draw up energy generation schedules and assess consumer demand to manage the work of Microgrid;

- ultra-short-term - for a period of several minutes to one hour, used to monitor the network in real time. 
When building forecast models, the main thing is to achieve high forecasting accuracy. This is influenced by the chosen forecasting technique, factors influencing the projected indicator, as well as the parameters of the constructed forecasting model. Models for forecasting the generation and consumption of electricity can be divided into multifactor models and models based on time series. Multifactor models are based on cause-and-effect relationships, and time series forecasting models use historical data over a period of time to predict. Time series forecasting models are divided into categories: statistical models, models based on machine learning, and hybrid models.

When optimizing the work of Microgrid, you should first formulate an optimization problem, which can be both multicriteria and meet such criteria:

- maximum output power at a given time;

- minimum operating costs;

- maximum cycle of operation of excess energy storage systems;

- minimum emissions into the environment, etc.

The following are the results of a study of models of short-term forecasting of electricity consumption from Microgrid. The analysis of factors influencing the need for electricity by different types of consumers and the categories of input data for the forecasting process:

- meteorological data - air temperature, humidity, wind speed, clouds, etc. ;

- electricity consumption - historical data of electricity consumption;

- calendar data - determine the seasonal factor;

- work schedule data - for enterprises that work according to the established schedule in one shift or in several shifts;

- other data that take into account the specifics of electricity consumption by a particular consumer.

The process of short-term forecasting in general consists of stages, which are shown in fig. 3.1. 


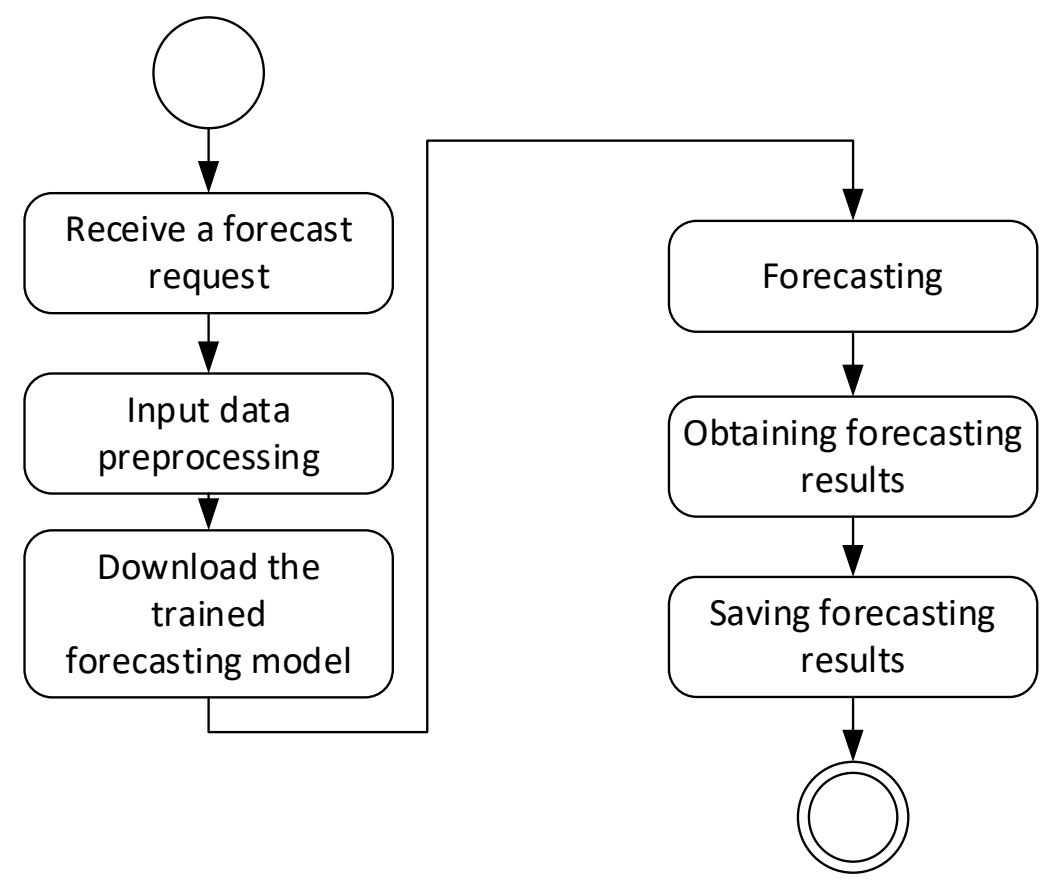

Fig. 3.1. Activity diagram of the short-term forecasting process

First it is necessary to generate input data, pre-process them. The stages of preprocessing are data cleaning from gaps, data normalization, and formation of an array of input data for the forecast model. To set the forecast input data, you need to download a previously built model that will adequately reproduce the need for electricity for future periods. When using the model, you need to calculate the prediction error, compare it with the allowable, and in case of exceeding the allowable value to make changes to the built model. Power consumption forecast can be performed both at the request of the user and automatically at set times. The prediction result must be saved in the database.

The peculiarity of forecasting as a scientific problem is that there is no single universal forecasting model. A forecast model should be built for each electricity consumption object using archival data of this object.

Analysis of literature studies has shown that the most commonly used methods for constructing prediction models are regression and autoregressive models ARMA, ARIMA, ARIMAX, SARIMAX, the method of constructing regression models using 
fuzzy logic, the method of reference vectors SVM, the method of exponential smoothing, theories intelligence.

Autoregressive forecasting methods are used when there is a linear relationship between the current value of the variable in the time series and past values. The use of the ARIMA model allows to build the predicted energy consumption of the model with high accuracy up to $97 \%$ with correctly selected regression model coefficients [12]. The SARIMAX model is used when seasonality is traced in the input data set, so it will give the most accurate results among autoregressive methods for forecasting electricity consumption. At the same time, the accuracy of the forecast model increases if not only parameters that directly affect electricity consumption, such as ambient temperature, but also the interaction of exogenous variables are used as input variables. [13].

The method of exponential smoothing is used to predict time series. The accuracy of forecasting when using this method depends on the choice of smoothing coefficients of the series, trend and seasonality, as well as the value of the data series. Analysis of the efficiency of the exponential smoothing method using power consumption data in the industrial sector showed from $81 \%$ to $96 \%$ accuracy for the model of linear exponential smoothing (Holt model), from 91\% to 97\% accuracy for the model with a damped trend depending on the data set [14]. When forecasting electricity consumption on the example of electricity consumption of university buildings, the forecasting accuracy for different types of models of exponential smoothing is approximately $88 \%$ [15].

Facebook's Prophet Library is also used to predict time series, which is based on a methodology for adjusting additive regression models, which uses functions that approximate the trend of the series, seasonal fluctuations and individual significant days, presented in the form of indicator variables. The result of comparing the accuracy of the Prophet model with the exponential smoothing model (Holt-Winters model) for long-term forecasting of electricity generation showed an accuracy above 95\% for both types of models, with the most accurate Holt-Winters model taking into account daily, weekly and annual seasonality. The use of the Prophet model for 
forecasting electricity consumption by household consumers, presented in [16], showed that the forecasting error can be up to $12.5 \%$, the accuracy of the model was influenced by the selection of parameters on which electricity consumption depends. The Prophet model does not establish a hidden relationship between power consumption and external factors, which reduces the accuracy of the forecast. The advantages of the Prophet model include less time to learn the model compared to machine learning methods.

Since the process of electricity consumption is characterized by uncertainty, there are peak values associated with various factors, an effective method of forecasting electricity consumption is to build a fuzzy regression model [17], which shows the best results for short-term forecasting. The advantages of this method include the ability to replace large sets of forecasting data with sets of fuzzy inference, the ability to formalize the experience of experts on energy fluctuations, the versatility of the method with minor modifications for forecasts of different time periods [18]. The application of this method for medium-term forecasting of electricity consumption showed high forecasting accuracy of 96-98\%, which has a slight deviation from the forecast values using ARIMA models and exponential smoothing [19].

Neural network models are widely used in forecasting power consumption, due to their ability to model nonlinear relationships between input and output variables, and allow to achieve high forecasting accuracy. Before using a neural network forecasting model, it is necessary to conduct its training, selecting the optimal parameters that will give the best forecasting accuracy. The learning process of the neural network in general is shown in Fig. 3.2 as a diagram of activity.

The most commonly used types of neural networks for prediction are direct propagation neural networks FFNN, nonlinear neural networks with exogenous NARX inputs, radial-based neural networks RBF, recurrent neural networks RNN, neural networks learning by the inverse propagation algorithm short-term LSTM memory. 
The vast majority of neural network models are effective if the inputs are properly selected and the models are configured. In [20], when using a neural network of the BPNN type for short-term prediction of power consumption, more than $90 \%$ is achieved, and it increases when the model of meteorological factors is included in the inputs. A study of the use of the NARX neural network for mediumterm prediction of power consumption conducted on a data set by the University of Malaysia showed that the accuracy for the NARX model is approximately $98 \%$, which is more accurate than for time series, fuzzy time series and multiple linear regressions [21].

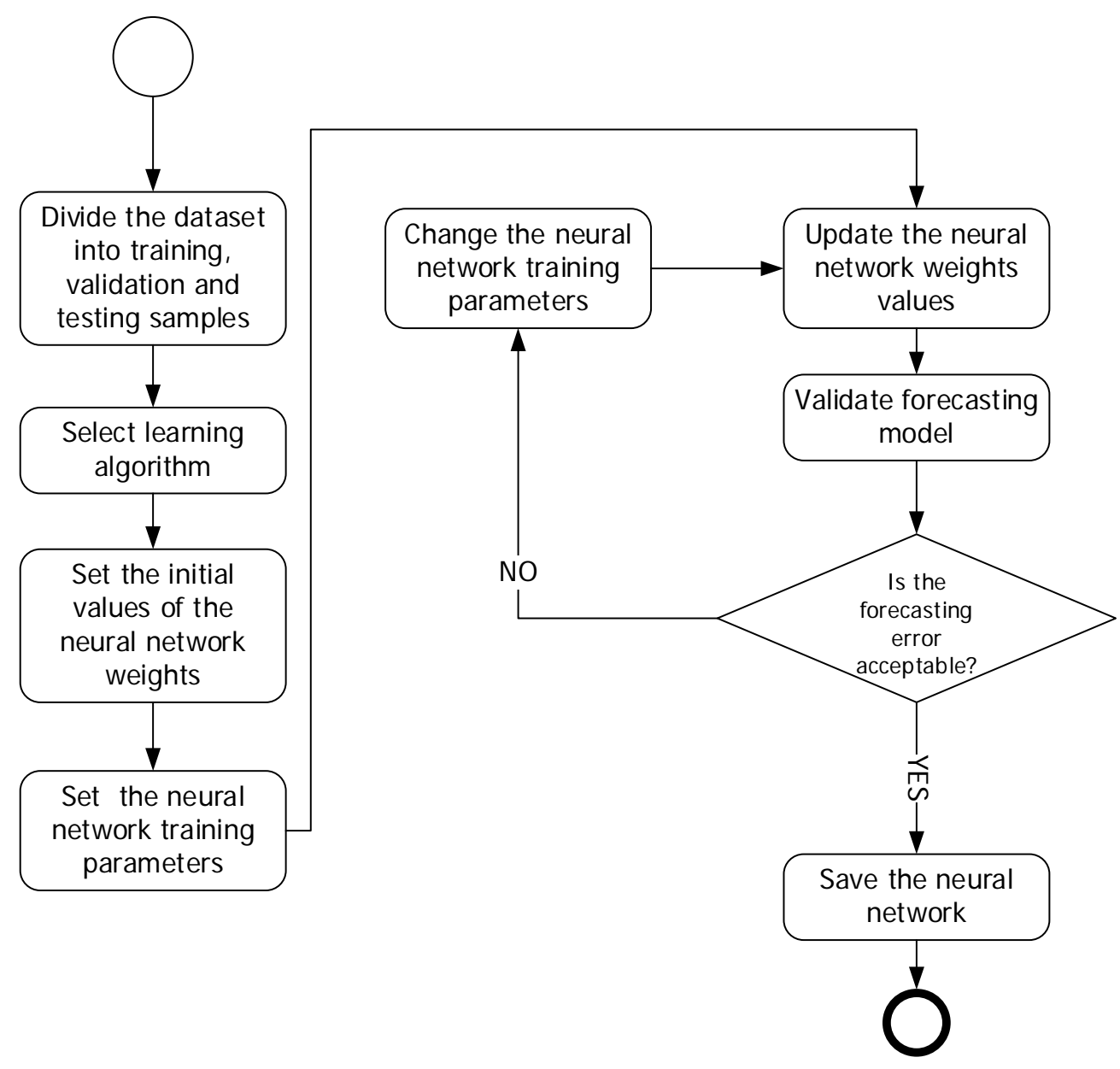

Fig. 3.2. Neural network training activity diagram for prediction

Among other types of neural networks, the LSTM model has advantages for short-term prediction of power consumption using data sets collected over a long period of time, as it can reproduce long-term time relationships in data sequences 
$[22,23]$. In comparison with NARX, SVM, SARIMA models for short-term forecasting of power consumption the LTSM model gives the best result [24].

Comparison of the accuracy of short-term forecasting models based on SARIMAX regression analysis, exponential smoothing, Prophet forecasting model and LTSM neural network was performed using archival data of electricity consumption at the Melitopol gas station for 2011-2013. Analysis of the time series of electricity consumption showed no trend and the presence of a seasonal component. There is an increase in electricity consumption in winter and at night. There is a correlation between the length of the day and electricity consumption, as well as the load of the gas station. The created data set for forecasting contains parameters - year, month, day of the week, hour of the day, the amount of electricity consumed for a period of one hour, the length of daylight, and the load of the gas station. The forecast is made every hour for a seven-day period.

To study the prediction accuracy of autoregressive models, the SARIMAX model was constructed with parameters $\mathrm{p}=0, \mathrm{~d}=1, \mathrm{q}=3, \mathrm{P}=3, \mathrm{D}=1, \mathrm{Q}=3, \mathrm{~m}=$ 12, using exogenous repressors: daylight length, load gas station. The model according to the best parameter of the information criterion Akaike AIC and Bayesian information criterion BIC, which are 86949.315 and 87127.726, respectively, was chosen. A recurrent neural network with long short-term LSTM memory was selected as the neural network for power consumption prediction. The LSTM model is constructed with the following parameters: 100 neurons in the LSTM layer, the Dense layer contains 1 neuron. The model includes an additional layer Dropout $=0.1$, which prevents retraining of the neural network. 20 epochs of neural network training were performed, the parameter batch_size, which determines the amount of training data for one pass of the model, is equal to 100 .

An additive exponential smoothing model for short-term power consumption forecasting was constructed using a seasonal period of 24. A Prophet forecasting model was also built using a library from Facebook.

All forecasting models are built in the Python environment using the appropriate libraries. A comparison of the accuracy of the developed models on a 
test sample of data for a calendar period of one week. The results of the calculated forecasting errors are given in table 3.1.

Table 3.1

Comparison of forecasting errors of developed models

\begin{tabular}{|c|c|c|c|}
\hline Model type & MAE, kWh & RMSE, kWh & MAPE, \% \\
\hline SARIMAX & 4.0 & 9.9 & 8.0 \\
\hline $\begin{array}{c}\text { Exponential model } \\
\text { smoothing }\end{array}$ & 4.0 & 11.8 & 7.8 \\
\hline Prophet & 4.0 & 11.8 & 7.8 \\
\hline LTSM & 2.6 & 3.4 & 5.1 \\
\hline
\end{tabular}

According to the results of the analysis of forecasting models, it can be noted that all of them have acceptable forecasting accuracy with a MAPE value of less than $10 \%$. The most accurate result among the developed forecasting models was shown by the neural network model LTSM, which confirms the effectiveness of machine learning models in short-term forecasting of electricity consumption.

The results of short-term forecasting, which determine the amount of electricity consumed for future periods of up to seven days, are used in the operational management of MicroGrid modes and are input to the management decision support system.

\section{MODELS OF DECISION SUPPORT IN ENERGY LIFECYCLE MANAGEMENT IN MICROGRID}

Today, different types of energy and different capacities can be used in one energy system, which emphasizes the importance of comparisons between alternative systems. Under these conditions, a comprehensive comparison between several energy systems is quite complex, requiring different knowledge bases, including technological, environmental, economic, social and political. In addition, different approaches can be used. 
The right decision to determine the priority list of possible alternative solutions among energy systems is important for the implementation of a successful project, because the wrong choice of alternative solution can lead to negative consequences in the environment, economy and society.

Fuzzy logic techniques are widely used to support decision-making in MicroGrid planning and operation management. A multicriteria method for selecting alternatives to the structure of a hybrid energy network with renewable energy sources, based on the evaluation of alternative structures using utility theory and using the method of expert evaluation to determine linguistic variable evaluation criteria, is proposed in [25]. Ranking of alternatives is carried out according to socioeconomic and energy efficient scenarios. [26] presents a multi-criteria decisionmaking model for the optimal structure of Microgrid based on the gray cumulative similarity theory, which selects the criteria from the categories of efficiency, reliability of the power supply system and environmental protection. The expert evaluation method was used to calculate the weights of the sub-criteria. If alternatives to the Microgrid configuration are identified, a hierarchy analysis method is used to evaluate them and select the best solution for different scenarios.

According to the above, it can be argued that:

1. There is uncertainty about the problem of making decisions with hybrid information, which should be properly addressed. These problems are primarily related to the diversity of input information and the large number of evaluation criteria.

2. Given that the ranking of energy systems is a promising activity, it is important to consider hybrid data with different types of uncertainties (quantitative and qualitative).

3. It is necessary to reliably determine the key criteria and their possible different weights. In the decision-making process, the general list of ranked energy systems is determined according to a set of criteria in environmental, economic, social and other aspects. The impact of each criterion in different scenarios on the overall stability of the system is 
different, which means that the weight of the criterion will be different in different scenarios.

This paper proposes a decision-making process that includes five stages (fig. 4.1): the process of preparation for the ranking of alternatives, the formation of a system of criteria, collection and processing of data for criterion evaluation, ranking of alternatives.

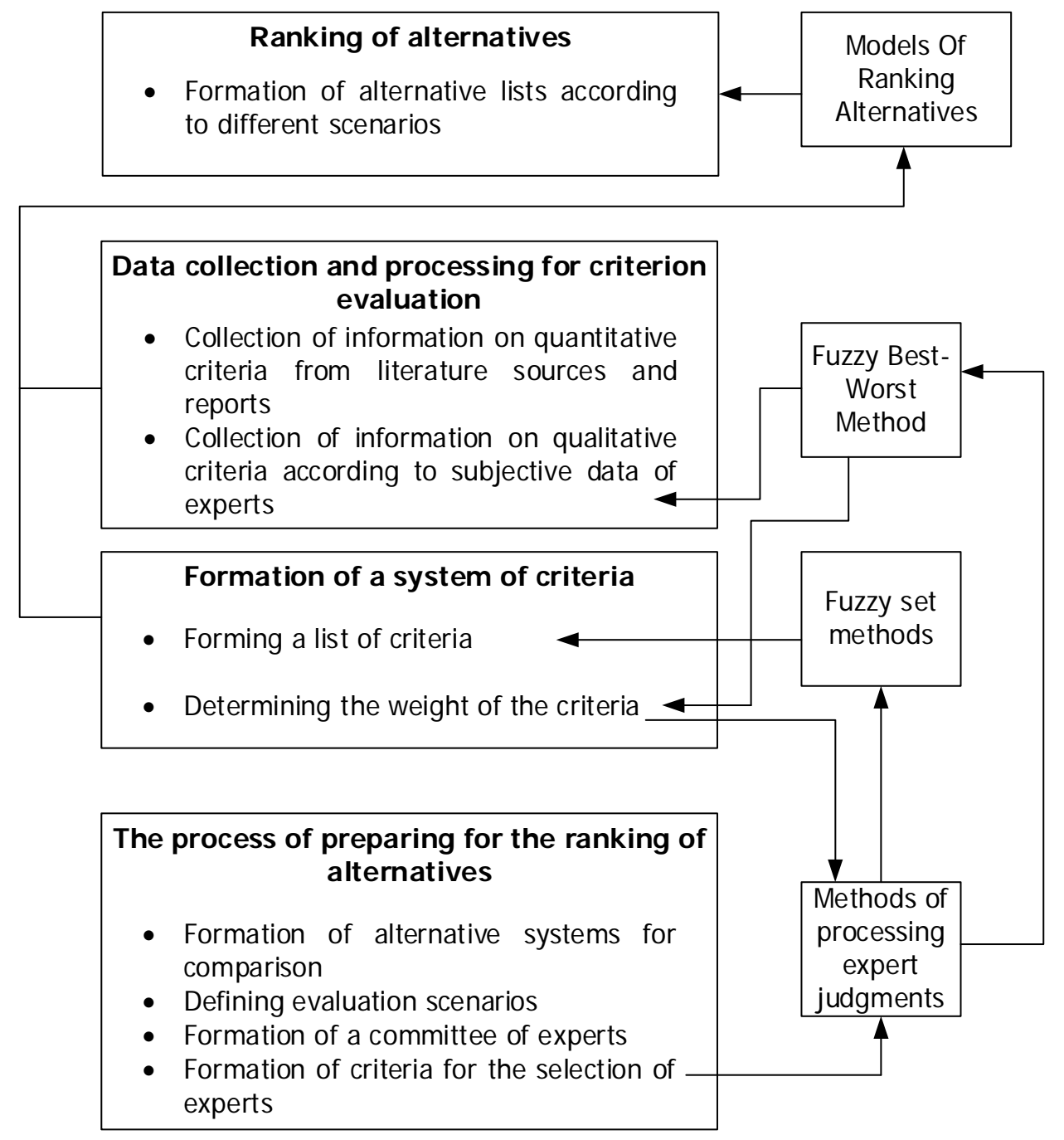

Fig. 4.1. Stages of the decision-making process to choose the optimal alternative solution

At the first stage, methods of processing experts' judgments are used to form a committee of experts and criteria for assessing the competencies of the experts themselves. 
In step 2, the fuzzy set method is used to filter the key criteria according to the experts' preferences for the importance of each criterion for a particular scenario. Step 3 uses the fuzzy best-worst method (FBWM) to convert the linguistic judgments of the experts into a consolidated weighing result. In step 4, FBWM is again used to convert subjective judgments about the effectiveness of qualitative criteria into numerical data. In step 5, alternative ranking models are used to determine the priority list.

A typical list of possible criteria for evaluating alternatives is presented in table 4.1.

Table 4.1

\section{List of evaluation criteria}

\begin{tabular}{|c|c|c|}
\hline Direction & Name & Type \\
\hline \multirow[t]{4}{*}{ Technological } & Energy efficiency & Quantitative \\
\hline & Effective system operating time & Quantitative \\
\hline & Scalability & Qualitative \\
\hline & Reliability & Qualitative \\
\hline \multirow{5}{*}{$\begin{array}{l}\text { Impact on the } \\
\text { environment }\end{array}$} & Depletion of the site by the resource is possible & Qualitative \\
\hline & Toxicity to humans & Qualitative \\
\hline & Impact on global warming & Qualitative \\
\hline & Land use & Qualitative \\
\hline & Waste generation & Qualitative \\
\hline \multirow[t]{3}{*}{ Economic } & Capital expenditures & Quantitative \\
\hline & Operating and maintenance costs & Quantitative \\
\hline & Possible sale & Qualitative \\
\hline \multirow[t]{2}{*}{ Social } & Social acceptance & Qualitative \\
\hline & Impact on public health & Qualitative \\
\hline \multicolumn{3}{|c|}{ OTHER } \\
\hline
\end{tabular}

Decision support for Microgrid network management can be formalized in the form of deterministic rules for regulating its operation, which are determined by the 
achievement of network performance indicators of the corresponding values [18]. These rules are transmitted to the controls in the form of control signals. They can be set as fuzzy inference rules that describe the production logic of Microgrid.

Rule-based Microgrid control, in contrast to optimization tasks, allows flexible response to changes in network modes and prevents the situation of insufficient power generation due to the implementation of management decisions, but performs too drastic changes in the state of the network. A sharp change in the parameters can cause additional peak loads due to overcharging of the storage device, which must be taken into account when formulating rules that describe the operation of the network.

\section{Conclusions}

The development of modern energy directly depends on the introduction of renewable energy sources into existing energy systems and the creation of Microgrid based on them. Widespread use in Microgrid based on renewable energy sources, such as solar panels and wind turbines, determines the dependence of energy production on climatic factors, so the task is to control Microgrid modes and, if necessary, switch to island mode, where Microgrid does not give excess energy to general network. At the same time in the management process provide maintenance of voltage stability and quality of electric energy, and also try to reduce expenses.

The energy management process is performed using appropriate information flows and models. Thus, the life cycle of energy in Microgrid is directly related to the life cycle of information and is provided by it.

Poor predictability of the level of generation from renewable energy sources requires improvement of forecasting models. Various statistical and probability models do not provide a sufficient level of forecasting. The study proved that the greatest difficulty for forecasting is short-term forecasting based on historical data over a fairly long period of time. All existing models in this case need additional adjustment. The analysis allowed us to identify the most promising models for forecasting. 
When determining the solution in the management of Microgrid there are problems of heterogeneity of input information and multicriteria. The proposed methods of overcoming diversity have proved their effectiveness and allowed to formulate new knowledge based on fuzzy rules used in the decision-making process.

\section{REFERENCES}

1. 1. Paris Agreement. [Online]. Available: https://zakon.rada.gov.ua/go/995_161

2. Hartono BS, Budiyanto, Rudy Setiabudy, "Review of Microgrid Technology", in Proceeding of 2013 International Conference on QiR, 2013, pp. 127-132, doi: 10.1109/QiR.2013.6632550.

3. D. Wang, J. Qiu, L. Reedman, K. Meng, L.L. Lai, "Two-stage energy management for networked microgrids with high renewable penetration", Appl. Energy 226, 2018, pp. 39-48, doi: http://dx.doi.org/10.1016/j.apenergy.2018. 05.112.

4. H. Krcmar, "Informationsmanagement," in Informationsmanagement,Springer Gabler, Berlin, Heidelberg, 2015, pp. 85-111.

5. T.Sachs, A.Gründler, M.Rusic, and G. Fridgen, "Framing microgrid design from a business and information systems engineering perspective", Business \& Information Systems Engineering, vol. 61(6), 2019, pp. 729-744.

6. M.Stadler, G.Cardoso, S.Mashayekh, T.Forget, N.DeForest, A.Agarwal, and A. Schönbein, "Value streams in microgrids: A literature review", Applied Energy, vol. 162, 2016, pp. 980 989.

7. Thomas Sachs, Anna Grundler, Milos Rusic, Gilbert Fridgen, "Framing Microgrid Design from a Business and Information Systems Engineering Perspective", Business \& Information Systems Engineering, 61, 2019, pp. 729-744, doi: 10.1007/s12599-018-00573-0.

8. Van-Hai Bui, Akhtar Hussain, Hak-Man Kim, and Yong-Hoon, "Optimal Energy Management of Building Microgrid Networks in Islanded Mode Considering Adjustable Power and Component Outages", Energies, 1, 2351, 2018, pp. 1-22, doi: 10.3390/en11092351.

9. Eun-Kyu Lee, Wenbo Shi, Rajit Gadh and Wooseong Kim, "Design and Implementation of a Microgrid Energy Management System", Sustainability, 8(11):1143, 2016, pp. 1-19, doi: 10.3390/su8111143.

10. Yimy E. Garcia Vera, Rodolfo Dufo-Lopez,Jose L. Bernal-Agustin, "Energy Management in Microgrids with Renewable Energy Sources: A Literature Review", Applied Sciences, 8(11):1143, 2019, pp. 1-28, doi: 10.3390/app9183854.

11. Mahmoud A. Hammad, Borut Jereb, Bojan Rosi, Dejan Dragan, "Methods and Models for Electric Load Forecasting: A Comprehensive Review", Logistics \& Sustainable Transport, vol. 11, no. 1, 2020, pp. 51-76, doi: 10.2478/jlst-2020-0004.

12. Junwei Miao, "The Energy Consumption Forecasting in China Based on ARIMA Model", in Proceedings of the International Conference on Materials Engineering and Information Technology Applications (MEITA 2015), pp. 192-196, 2015, doi: 10.2991/meita-15.2015.37.

13. Niematallah Elamin, MototsuguFukushige, "Modeling and Forecasting Hourly Electricity Demand by SARIMAX with Interactions", Energy, 165, 2018, pp. 257-268, doi: 10.1016/j.energy.2018.09.157. 
14. Muslima Zahan, Ron S. Kenett, "Modeling and Forecasting Energy Consumption in the Manufacturing Industry in South Asia", International Journal of Energy Economics and Policy, vol. 3, no. 1, 2013, pp. 87-98.

15. Y.W. Lee, K.G. Tay, Y.Y. Choy, "Forecasting Electricity Consumption Using Time Series Model", International Journal of Engineering \& Technology, 7(4), 2018, pp. 218-223, doi: 10.14419/ijet.v7i4.30.22124.

16. Alex Leung, "A Multivariate Model for Electricity Demand using Facebook Prophet". [Online]. Available: https://alex-leung.medium.com/a-multivariate-model-for-electricitydemand-using-facebook-prophet-f848ad1c9d77.

17. Sergii Tymchuk, Sergii Shendryk, Vira Shendryk, Ivan Abramenko and Anastasiia Kazlauskaite. "The Methodology of Obtaining Power Consumption Fuzzy Predictive Model for Enterprises", Advances in Design, Simulation and Manufacturing, pp. 210-219, 2020. DOI: 10.1007/978-3-030-50794-7_21.

18. Sebastien Bissey, Sebastien Jacques, and Jean-Charles Le Bunetel, "The Fuzzy Logic Method to Efficiently Optimize Electricity Consumption in Individual Housing", Energies, 10(11), 1701, 2017, pp. 1-22, doi: 10.3390/en10111701.

19. Pawel Pelka, and Grzegorz Dudek, " Prediction of monthly electric energy consumption using pattern-based fuzzy nearest neighbour regression", in Proc. Of ITM Web of Conferences, 15, 02005, 2017, doi: 10.1051/itmconf/20171502005.

20. Hao Song, Yu Chen, Ning Zhou, Genshe Chen, "Electricity consumption forecasting for smart grid using the multi-factor back-propagation neural network", in Proc. SPIE 11017, Sensors and Systems for Space Applications XII, 110170O, 2019, doi: 10.1117/12.2519058.

21. K. G. Tay, Hassan Muwafaq, Shuhaida Binti Ismail, Pauline Ong, " Electricity Consumption Forecasting Using Nonlinear Autoregressive with External (Exogeneous) Input Neural Network", Universal Journal of Electrical and Electronic Engineering, 6(5B), pp. 26-36, 2019. - DOI: 10.13189/ujeee.2019.061 605.

22. W. Kong, Z. Y. Dong, Y. Jia, D. J. Hill, Y. Xu and Y. Zhang, "Short-Term Residential Load Forecasting Based on LSTM Recurrent Neural Network," in IEEE Transactions on Smart Grid, vol. 10, no. 1, pp. 841-851, 2019, doi: 10.1109/TSG.2017.2753802.

23. Mesa Jiménez, J J; Stokes, L; Moss, C; Yang, Q; Livina, V N, "Modelling energy demand response using long short-term memory neural networks", Energy Efficiency, 13(6), pp. 12631280, 2020, doi: 10.1007/s12053-020-09879-Z.

24. Jian Zheng, Cencen Xu, Ziang Zhang and Xiaohua Li, "Electric load forecasting in smart grids using Long-Short-Term-Memory based Recurrent Neural Network," 2017 51st Annual Conference on Information Sciences and Systems (CISS), 2017, pp. 1-6, doi: 10.1109/CISS.2017.7926112.

25. Shulyma, O., Shendryk, V.,Parfenenko, Y., Shendryk, S., "The Model for Decision Support on Design of the Hybrid Renewable Energy System", in Proceedings of the 2017 IEEE 9th International Conference on Intelligent Data Acquisition and Advanced Computing Systems: Technology and Applications, IDAACS 2017, 2017, pp. 47-50, doi: 10.1109/IDAACS.2017.8095047

26. Sébastien Bissey, Sébastien Jacques and Jean-Charles Le Bunetel, "Selecting the Optimal Micro-Grid Planning Program Using a Novel Multi-Criteria Decision Making Model Based on Grey Cumulative Prospect Theory", Energies, 11, pp. 1-24, 2018, doi: 10.3390/en11071840. 